\title{
A note on the form of the psychophysical function near threshold
}

\author{
ROBERT F. FAGOT \\ University of Oregon, Eugene, Oregon 97403
}

\begin{abstract}
In many psychophysical scaling studies, a threshold parameter has been introduced into the power function to account for discrepancies from the simple power function at low intensities. Two such modifications have received special attention: the $\phi$ form, which involves translation on the intensity continuum; and the $\psi$ form, which involves translation on the psychological continuum. Marks and Stevens (1968) reviewed various proposed modifications of the power function and discussed methodological problems involved in discriminating between various forms by means of empirical tests. This brief paper is a reply to some of the methodological points made by Marks and Stevens and concludes that the evidence favoring a choice of the $\psi$ form over the $\phi$ form is more convincing than admitted by Marks and Stevens.
\end{abstract}

It has long been noted that the simple power function does not fit well for low intensities. To account for such discrepancies, a threshold parameter has been introduced into the power function in various ways, the following having received considerable attention:

$$
\begin{gathered}
\Psi=\mathrm{a}\left(\Phi \mathrm{k}-\mathrm{t}^{\mathrm{k}}\right) \\
\Psi=\mathrm{a}^{\prime}\left(\Phi-\mathrm{t}^{\prime}\right) \mathrm{m},
\end{gathered}
$$

where $\psi$ is psychological magnitude, $\Phi$ is physical intensity, and $t$ and $t^{\prime}$ are usually interpreted as threshold parameters (not necessarily equal if estimated indirectly in the scaling experiment). Equation 1 involves translation on the psychological continuum-the $\Psi$ form-and Equation 2 involves translation on the intensity continuum-the $\Phi$ form. Apparently, Equation 1 was first used by Stevens and Davis (1938) and McGill (1960), and a methodological study was reported by Fagot (1966). Equation 2 appears to have been formulated first by Ekman (1956) and later by Stevens (1959).

Marks and Stevens (1968) reviewed the various proposed modifications of the power function and related issues, focusing on Equations 1 and 2. This brief paper is a reply to some of the methodological points made by Marks and Stevens relative to a choice between Equations 1 and 2.

Marks and Stevens state that the issue of the appropriate form of the power function cannot be resolved by appeal to goodness-of-fit tests based on a few experiments. Their point is directed mainly toward the issue of biasing factors in the design of the experiments, but it should be pointed out that the evidence is perhaps somehwat more substantial than implied by this statement. Thus the data from each of the following studies were interpreted as providing a better fit to the $\Psi$ form than the $\Phi$ form: Zwislocki and
Hellman (1960) for various sensory modalities; the Lochner and Burger (1961) analysis of the Hellman and Zwislocki (1961) loudness data; McGill (1960) for individual loudness functions; Jameson (1965); Stewart, Fagot, and Eskildsen (1967); and Fagot and Stewart (1968) for brightness; Ekman (1961) and Ekman and Akesson (1965) for gustatory intensity; and Irwin and Corballis (1968) for loudness and softness. The last study is particularly interesting, in that the conclusion was not based on goodness-of-fit tests but on the finding that the $\Psi$ form produced functions in which loudness and softness were reciprocally related, whereas the $\Phi$ form produced a softness function that was almost three times as steep as the loudness function.

Numerous studies have been carried out in which the $\Phi$ form has been fitted to data but studies in which the $\Phi$ form was found to fit the data better than the $\Psi$ form are notably lacking. Ekman and Gustafsson (1967), as pointed out by Marks and Stevens, concluded that the $\Psi$ form did not provide as good a fit as the $\Phi$ form, but the basis for this conclusion is puzzling since the $\Phi$ form exponent was almost unity (1.003). In any event, the comparative studies that have been reported point to a better fit to the $\Psi$ form for a variety of continua.

Marks and Stevens advise caution in accepting the results of goodness-of-fit tests favoring a particular form of the power function due to the possible biasing effect of factors such as the choice of method and location of standard. They were critical of the experiment by Stewart, Fagot, and Eskildsen (1967) because the method of fractionation was used, asserting that this method was bound to favor the $\psi$ form over the $\Phi$ form. Marks and Stevens offer no real explanation for this puzzling conclusion, nor can any adequate basis be found in Stevens (1962), the source referred to by Marks and Stevens. In any event, if indeed the statement were true, surely it would lend support to the contention that the $\Psi$ form is a more 
appropriate form of the power function. Considering that there are two main classes of direct ratio scaling procedures to which the power function is applied (numerical response methods, such as magnitude and ratio estimation, and operation or production methods, such as fractionation), the Marks and Stevens assertion appears to concede that the only open question is, "which function best fits numerical response methods?"

Marks and Stevens were also properly concerned about the possible disruptive effects of location of standard on the problem of comparing the two forms of the power function. The experiment of Fagot and Stewart (1968) was criticized on this basis because the standard was the brightest stimulus in the set, and such an arrangement should produce brightness contrast. However, if one notes that the threshold in both forms is treated as a parameter and not a random variable, the basis for choice of standard should be clear: The brightest stimulus was chosen as standard so that the effective threshold would be relatively constant for all judgment trials. Ekman and Gustafsson (1967), the one study cited by Marks and Stevens as not providing a good fit to the $\Psi$ form, used the same procedure for the same reason.

Conceding that factors such as choice of standard may be disruptive and, hence, produce systematic errors, it is certainly not obvious that such disruption should favor one form of the power function over another. For example, in the experiment of Fagot and Stewart (1968) in which the potentially disruptive high standard was used, three of the subjects showed marked systematic errors for both forms, consistent with the interpretation of the high standard as a disruptive factor. However, the analysis of variance tests for individual subjects showed that the deviations mean square (MSQD) was smaller for all subjects for the $\Psi$ form. In fact, the superiority of the $\Psi$ form appeared to be even greater for the three subjects not exhibiting a disruptive effect due to high standard: Average MSQD for the $\Phi$ form was 1.74 times that for the $\Psi$ form for the three subjects showing the disruptive effect and 2.26 times for those not showing the effect.

It should also be noted that the studies cited in support of the $\Psi$ form do not depend on any one scaling method or choice of standard. They involve a diversity of perceptual attributes; several scaling methods, such as fractionation, magnitude estimation, and ratio estimation; and a variety of stimulus presentations (all pairs, standard in middle, at lower end, and at upper end). Regardless of perceptual attribute, scaling method, or stimulus presentation, these studies have concluded in favor of the $\Psi$ form of the power function.

Marks and Stevens argue against the feasibility of deciding empirically between the two forms of the power function, due in part to lack of precision in scaling techniques. This implies that the experiments conducted to discriminate between the two forms had low statistical power. Yet, a substantial number of studies reported positive results in favor of the $\Psi$ form, a conclusion that should be interpreted as even more compelling in view of the lack of precision in scaling techniques and consequent low power.

As a final point, contrary to the concern of Marks and Stevens relating to potentially disruptive factors in psychophysical experiments, it could be argued that if the power function is to maintain the status of an important generalization, then it must account for the effects of variation in important independent variables, such as scaling method, location of standard, and range (see Poulton, 1968). Hence, rather than avoiding particular scaling methods and procedural variations that introduce systematic errors, the emphasis should be on complicating the model (psychophysical function) to account for such deviations (see, for example, Teghtsoonian, 1973).

\section{REFERENCES}

EKMAN. G. Subjective power functions and the method of fractionation. Report from Psychology Laboratory, University of Stockholm, 1956, No. 34.

Ekman, G. Methodological note on scales of gustatory intensity. Scandinavian Journal of Psychology, 1961, 2, 185-190.

Ekman, G., \& Ákesson, C. Saltness, sweetness, and preference. Scandinavian Journal of Psychology, 1965, 6, 241-253.

Ekman, G., \& Gustafsson, U. The absolute threshold and the psychophysical function in brightness vision. Report from Psychology Laboratory, University of Stockholm, 1967, No. 233.

FAGOT, R. F. Alternative power laws for ratio scaling. Psychometrika, 1966, 31, 201-214.

Fagot, R. F., \& Stewart, M. R. An experimental comparison of stimulus and response translated power functions. Perception \& Psychophysics, 1968, 3, 297-305.

Hellman, R. P., \& Zwislocki, J. Some factors affecting the estimation of loudness. Journal of the Acoustical Society of America, 1961, 33, 687-694.

Irwin, R. J., \& Corballis, M. C. On the general form of Stevens' Law for loudness and softness. Perception \& Psychophysics, 1968, 3, 137-144.

JAMESON, D. Threshold and supra-threshold relations in vision. Proceedings of the International Colour Meeting, Lucerne. $1965,1,128-136$.

Lochner, J. P. A., \& Burger, J. F. Form of the loudness function in the presence of masking noise. Journal of the Acoustical Society of America, 1961, 33, 1705-1707.

Marks, L. E., \& Stevens, J. C. The form of the psychophysical function near threshold. Perception \& Psychophysics, 1968, 4, 315-318.

McGill, W. A. The slope of the loudness function: A puzzle. In H. Gulliksen \& S. Messick (Eds.) Psychological Scaling: Theory and applications. New York: Wiley, 1960. Pp. 67-81.

Poulton, E. C. The new psychophysics: Six models for magnitude estimation. Psychological Bulletin, 1968, 69, 1-19.

Stevens, S. S. Tactile vibration: Dynamics of sensory intensity. Journal of Experimental Psychology, 1959, 59, 210-218.

Stevens, S. S. The surprising simplicity of sensory metrics. American Psychologist, 1962, 17, 29-39.

Stevens, S. S., \& Davis, H. Hearing: Its psychology and physiology. New York: Wiley, 1938.

Stew art, M. R., Fagot, R. F., \& Eskildsen, P. R. Invariance tests for bisection and fractionation scaling. Perception \& Psychophysics, 1967, 2, 323-327.

TEghtsoonian, R. Range effects in psychophysical scaling and a 
revision of Stevens' law. American Journal of Psychology, 1973, 86. 3-27.

Zwislocki. J., \& Hellman, R. On the psychophysical law.
Journal of the Acoustical Society of America, 1960, 32, 924.

(Received for publication August 4, 1975.) 